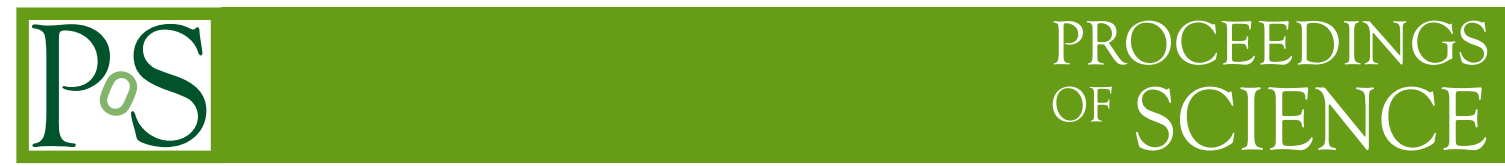

\title{
Nuclear Physics at the Lanzhou Storage Ring
}

\author{
M.Wang, H.S.Xu, Y.H.Zhang, X.L.Tu, X.W.Ma \\ Institute of Modern Physics, CAS, 730000 Lanzhou, China
}

\begin{abstract}
Since the last conference stori' 08 , progresses have been made at the Lanzhou storage ring system HIRFL-CSR. The present status of physics research activities in different experimental setups at CSR is introduced. Mass measurement of short-lived nuclei at CSRe is the main project carried out at present. Two experiments of mass measurements were performed using ${ }^{78} \mathrm{Kr}$ and ${ }^{58} \mathrm{Ni}$ as primary beams. The consequence of newly measured masses is discussed.
\end{abstract}

8th International Conference on Nuclear Physics at Storage Rings-Stori11,

October 9-14, 2011

Laboratori Nazionali di Frascati dell'INFN, Italy

\footnotetext{
*Corresponding E-mail: hushan@impcas.ac.cn
} 


\section{Introduction}

In 2008, when the 7th International Conference on Nuclear Physics at Storage Rings (Stori'08) was held at Lanzhou, the Cooling Storage Ring (CSR) was just under commissioning [1]. The CSR complex [2], consisting of a main ring (CSRm), an experimental ring (CSRe), and a radioactive beam line (RIBLL2) connecting the two rings, is the post-acceleration system of the Heavy Ion Research Facility in Lanzhou (HIRFL). The two formerly existing cyclotrons SFC ( $K=69)$ and SSC $(K=450)$ of the HIRFL are used as its injector system.

With these machines, all stable nuclides, from proton to uranium, can be provided at different energies for different researches. The ${ }^{12} \mathrm{C}$ beam with maximum energy of $1.1 \mathrm{GeV} / \mathrm{u}$ was delivered at CSRm. Radioactive ion beams (RIB) are provided via projectile fragmentation at the two secondary beam lines: RIBs of tens of MeV/u are delivered at RIBLL1, and RIBs of hundreds of $\mathrm{MeV} / \mathrm{u}$ are delivered at RIBLL2.

\section{Experiments at CSR}

The beams extracted from CSRm can be used for cancer therapy and many physics experiments [3], as shown in Fig.1. At the cancer therapy station, 45 patients with deeply seated tumor have been treated successfully with carbon beam from CSRm. PISA [4], which was once at the COSY ring for proton induced spallation study, has been shipped to Lanzhou in 2007 and reassembled at an experimental terminal of CSRm. A test experiment at PISA is planned to be carried out in near future.

\subsection{The Hadron Physics Lanzhou Spectrometer}

At CSRm, an internal target experiment is planned for hadron physics, which focuses on hadron spectroscopy, polarized strangeness production and medium effect. Proton beam and a pellet proton target will be used for this purpose. The spectrometer's name is HPLUS from Hadron Physics LanzhoU Spectrometer [5]. Based on Pluto++, Pythia and phase space calculation, the conceptual design of the spectrometer is now fixed. We are working to push this project to the final approval.

\subsection{The external target facility}

The external target facility (EFT) of CSRm is located at a terminal of the RIBLL2 line. Stable beams or radioactive ion beams via projectile fragmentation can be used there, by using slow extraction or fast extraction from CSRm. The EFT detection system consists of a large acceptance dipole, wire chambers combined with a ToF wall for charged particle detection, and a neutron wall for neutron detection. One of the physics goals at EFT is to study symmetry energy of high density nuclear matter [6]. $\pi+/ \pi$ - is a sensitive probe for that research. Recently a test experiment has been carried out at ETF. ${ }^{12} \mathrm{C}$ beams at $200 \mathrm{MeV} / \mathrm{u}$ and $600 \mathrm{MeV} / \mathrm{u}$ and a natural carbon target were used in this experiment. Pions were produced and $\pi+$ and $\pi$ - could be clearly identified from the particle identification scattering plot. The $\pi+/ \pi$ - ratio is around 1 from the preliminary result. Although much work needs to be improved, the present experiment constitutes a good starting point for further study. 


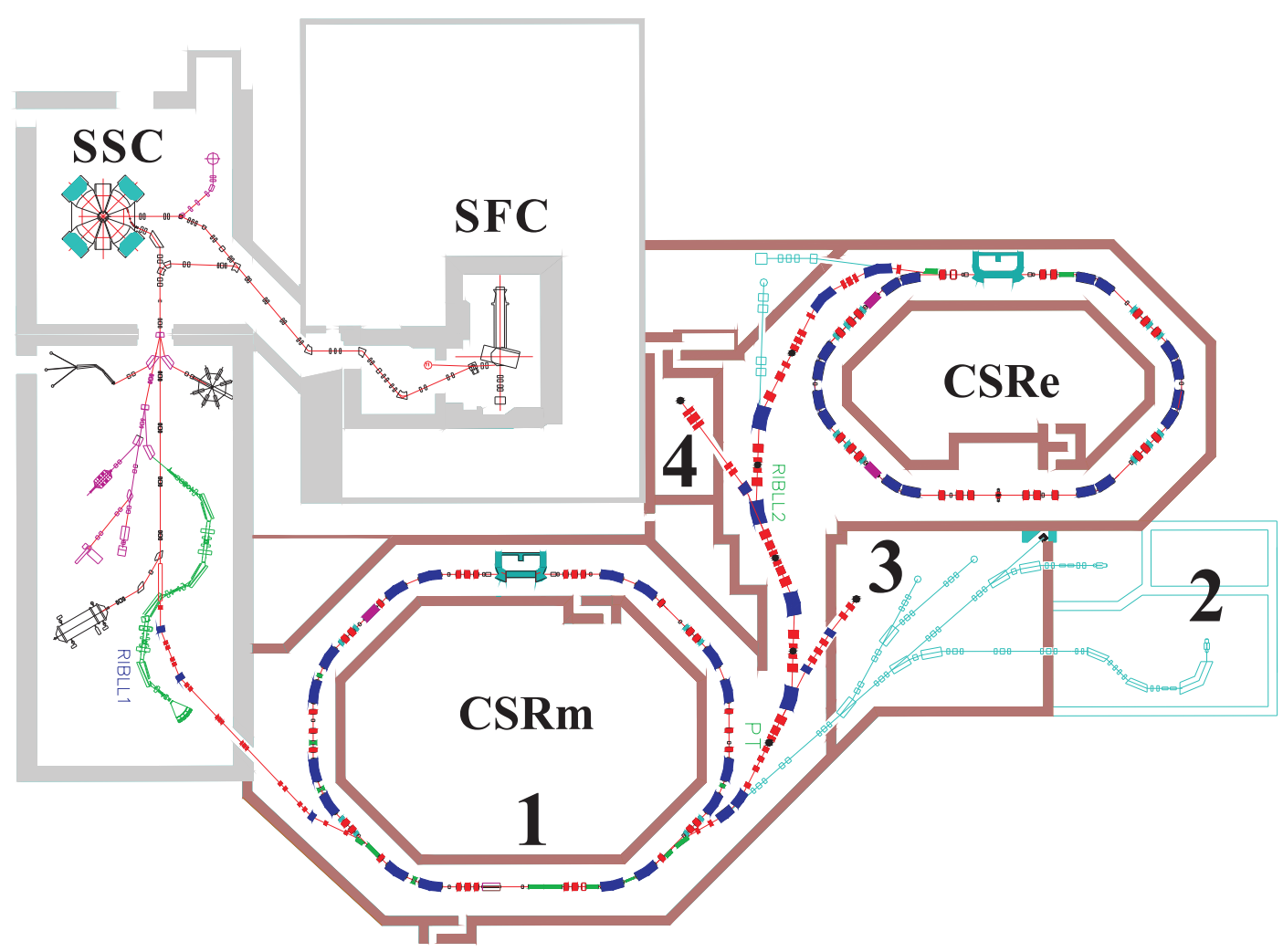

Figure 1: Schematic overview of HIRFL-CSR and some experimental areas: 1. HPLUS; 2. tumor therapy station; 3. PISA; 4. EFT.

\subsection{The CSRe ring}

High precision mass measurements and decay studies can be carried out at the CSR experimental ring CSRe. In addition to the nuclear physics, atomic physics programs, including X-ray spectroscopy, dielectronic recombination and so on, are also planned [7]. Recently a test experiment has been performed at the internal gas target station of CSRe. In this experiment, fully stripped Xe beam was stored in the ring successfully and nitrogen gas target worked well. X-ray spectra at different angles were measured. Nuclear physics experiments, such as elastic scattering and transfer reactions, can also be performed here in future research.

\section{Mass measurement of short-lived nucei}

Nuclear mass is a fundamental property of the nucleus. It reflects the complex interplay of strong, weak and electromagnetic interactions in a nucleus. Nuclear masses play an important role in nuclear structure, nuclear astrophysics, and represent a test for standard model and fundamental symmetries. One of the advantages of the use of storage rings as mass spectrometry is that many nuclei can be easily stored simultaneously so that a relatively large mass distribution can be covered in one run and correlations between the nuclei can be established. 


\subsection{Principle of mass measurement at CSRe}

The revolution times $\mathrm{T}$ of various ions in a storage ring are related (in first order) to their mass-to-charge ratios $\mathrm{m} / \mathrm{q}$ via

$$
\frac{\Delta T}{T}=\frac{1}{\gamma_{t}^{2}} \frac{\Delta(m / q)}{(m / q)}-\left(1-\frac{\gamma^{2}}{\gamma_{t}^{2}}\right) \frac{\Delta v}{v},
$$

where $\gamma$ is the relativistic Lorentz factor and $\gamma_{t}$ denotes the transition point of the storage ring [8]. So the $\mathrm{m} / \mathrm{q}$ can be determined if the revolution times of the ions are measured and the second term that contains the ion velocity spreads, $\Delta \mathrm{v} / \mathrm{v}$ is minimized. Two methods are adopted for this purpose. In the first method the velocity spread $\Delta \mathrm{v} / \mathrm{v}$ is reduced to the order of $10^{-7}$ by cooling the beam and the revolution frequencies of the stored ions are measured by means of a Schottky detector. This method is called Schottky Mass Spectrometry (SMS). In the second method $\gamma$ of the ions of interest is set as close as possible to the $\gamma_{t}$ by tuning the storage ring in isochronous mode. This makes the ions of one species with different velocities to circulate in the ring with an identical revolution period. Since cooling of the stored ions is not necessary, the IMS is suitable for the mass measurements of shot-lived nuclei with half-lives of several tens of microseconds.

A test run for mass measurement was carried out at CSRe at the end of 2007 [9]. The isochronous mass spectrometry was realized and a resolving power $\mathrm{m} / \Delta \mathrm{m}$ of 100,000 was achieved in the test experiment. Encouraged by that result, new experiments were performed and at present mass measurements of short-lived nuclide become the main physics project at CSRe.

\subsection{Experiment with ${ }^{78} \mathrm{Kr}$}

Main goal of the first experiment is the measurement of the masses of short-lived $A=2 Z-1$ nuclei ${ }^{63} \mathrm{Ge},{ }^{65} \mathrm{As},{ }^{67} \mathrm{Se}$, and ${ }^{71} \mathrm{Kr} .{ }^{78} \mathrm{Kr}$ was used as primary beam in this experiment. The ${ }^{78} \mathrm{Kr}{ }^{19+}$ beam of $4 \mathrm{MeV} / \mathrm{u}$ provided by SFC was stripped to $28+$ by a carbon foil placed inside the beam line, and then injected into CSRm. The ${ }^{78} \mathrm{Kr}$ ions were accumulated in CSRm and then accelerated to $483.4 \mathrm{MeV} / \mathrm{u}$. A beam intensity of up to $10^{8}$ particles per spill has been achieved. The highenergy ${ }^{78} \mathrm{Kr}$ beam was fast extracted from the CSRm and focused upon a $15 \mathrm{~mm}$ thick beryllium production target at the entrance of the in-flight fragment separator (RIBLL2). The exotic nuclei were produced by projectile fragmentation of ${ }^{78} \mathrm{Kr}$ and emerged from the target predominantly as bare ions. After in-flight separation of the fragments in RIBLL2, the cocktail beam of exotic nuclei was injected into CSRe. RIBLL2 and CSRe were set to a magnetic rigidity of $\mathrm{B} \rho=5.9493 \mathrm{Tm}$ to optimize the transmission of the $A=2 Z-1$ nuclei. Other nuclides within the acceptance of the RIBLL2-CSRe system were also transmitted and stored in CSRe. After each injection, about 20 ions were simultaneously stored in CSRe.

The revolution times were measured using a timing detector [10] equipped with a $19 \mu \mathrm{g} / \mathrm{cm}^{2}$ thin carbon foil of $40 \mathrm{~mm}$ in diameter. Each stored ion passed through the timing detector at every revolution in CSRe. Secondary electrons released from the foil due to the interaction with the ions were guided isochronously by perpendicularly arranged electrostatic and magnetic fields to a set of microchannel plates. The time resolution of the detector was about $50 \mathrm{ps}$, and the detection efficiency varied from $20 \%$ to $70 \%$ depending on the ion type and the number of the ions stored. The signals from the detector were sampled with a digital oscilloscope Tektronix DPO 71254 at a 
sampling rate of $50 \mathrm{GHz}$. The recording time was $200 \mu$ s for each injection corresponding to $\sim 320$ revolutions.

The resolving power of CSRe mass spectrometry is mainly determined by instabilities of the magnetic fields. These instabilities cause small shifts in the revolution time of the entire spectrum for different injections. In order to reduce the effect of these shifts, we measured the revolution time of each particle relative to the revolution time of a selected reference nuclide present in the same injection. As not all the nuclei were present in each injection, we used seven reference peaks $\left({ }^{19} \mathrm{Ne}\right.$, ${ }^{21} \mathrm{Na},{ }^{23} \mathrm{Mg},{ }^{25} \mathrm{Al},{ }^{27} \mathrm{Si},{ }^{29} \mathrm{P}$, and ${ }^{31} \mathrm{~S}$ ) creating seven independent spectra that contained $69 \%$ of all particles. The resolving power of the shift-corrected spectra is 170,000 . The seven subspectra and an integral spectrum have been analyzed separately and provided consistent results. Figure 2 presents the histogram of the extracted revolution times for a part of the $A=2 Z-1$ nuclei. Particle identification was realized by comparing the measured revolution-time spectrum with a simulated one. All the $A=2 Z-1$ nuclei with experimentally known masses from ${ }^{33} \mathrm{Cl}$ through ${ }^{61} \mathrm{Ga}$ (except for ${ }^{43} \mathrm{Ti}$ since it has a low-lying isomeric state) were used to fit $\mathrm{m} / \mathrm{q}$ versus $\mathrm{T}$ employing a second order polynomial. The ME values of ${ }^{63} \mathrm{Ge},{ }^{65} \mathrm{As},{ }^{67} \mathrm{Se}$, and ${ }^{71} \mathrm{Kr}$ were obtained by extrapolating the fit function to the corresponding T. In order to estimate possible systematic errors, the literature ME values have been redetermined in the analysis using a different number of reference nuclei and different extrapolation ranges. The errors for the new masses are dominated by statistical errors. Details of the data analysis are described in [11].

\subsection{Experiment with ${ }^{58} \mathrm{Ni}$}

In another experiment, ${ }^{58} \mathrm{Ni}$ beam was used as the primary beam. Similar to the first experiment described above, the ${ }^{58} \mathrm{Ni}$ beam was accumulated in CSRm and then accelerated to an energy of $463 \mathrm{MeV} / \mathrm{u}$. The high-energy beam was fast extracted from CSRm and focused upon a $15 \mathrm{~mm}$ thick beryllium production target. The exotic nuclei of interest were produced by projectile fragmentation of ${ }^{58} \mathrm{Ni}$. After in-flight separation of the fragments in RIBLL2, the cocktail beam of exotic nuclei was injected into CSRe for mass measurements. The magnetic rigidity of the ring was set to ${ }^{47} \mathrm{Mn}$.

In this experiment, the time stability of the magnetic field was better than the first experiment, which represented about $10^{-5}$, thanks to an update of the power supply system of CSRe. The data analysis method of using relative revolution frequencies was not necessary this time. The integral spectrum of revolution times is shown in Fig. 3.

\subsection{Discussion of the experimental results}

In the two experiments, the masses of 10 nuclei have been measured for the first time in CSRe, and 10 other nuclei have been remeasured with higher accuracy. Some of the nuclei are located on the path of the rapid proton capture process which is believed to power the type I X-ray bursts. ${ }^{64} \mathrm{Ge}$ is a potentially important waiting point along rp-process because of its long half life. The effective lifetime of ${ }^{64} \mathrm{Ge}$ in the stellar environment is sensibly related to the proton separation energy of ${ }^{65} \mathrm{As}$. By measuring the mass of ${ }^{65} \mathrm{As}$ in our experiment, the proton separation energy for ${ }^{65} \mathrm{As}$ is deduced to be $-90(85) \mathrm{keV}$. X-ray burst model calculations with the new mass excess of ${ }^{65} \mathrm{As}$ suggest that the majority of the reaction flow passes through ${ }^{64} \mathrm{Ge}$ via proton capture, indicating 


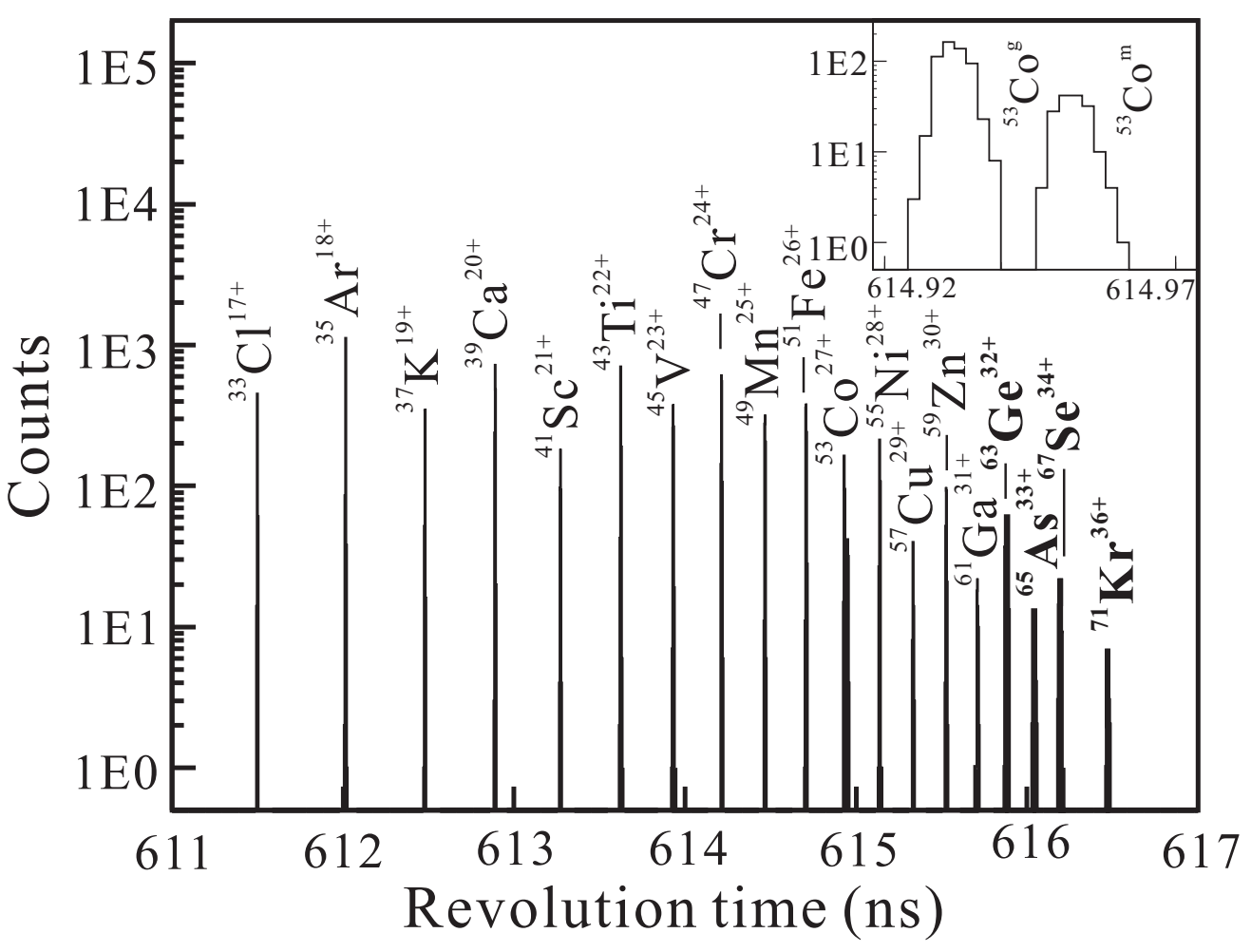

Figure 2: The revolution time spectrum obtained in ${ }^{78} \mathrm{Kr}$ experiment. The insert shows the well-resolved ground and isomeric $\left(\mathrm{T}_{1 / 2}=247 \mathrm{~ms}, E^{*}=3.197 \mathrm{MeV}\right)$ states of ${ }^{53} \mathrm{Co}$.

that ${ }^{64} \mathrm{Ge}$ is not a significant rp-process waiting point [12]. Other nuclei such as ${ }^{43} \mathrm{~V}$ and ${ }^{47} \mathrm{Mn}$ are located on the path of rp-process and their impact on astrophysics calculation will be studied.

With the newly measured masses of the $A=2 Z-3$ nuclei, all members of several $\mathrm{T}=3 / 2$ quartets are now known experimentally. This provides the opportunity to test the isobaric multiplet mass equation, which is of fundamental importance of isospin symmetry in nuclear physics. This work is ongoing.

\section{Perspective}

To realize SMS at CSRe, the Schottky pick-up should be sensitive to a single ion circulating in the ring. The former Schottky pick-up at CSRe did not fulfill the requirement. A fast and sensitive resonant Schottky pick-up [13], which is the same as used at ESR, has been built at GSI and shipped to Lanzhou early 2011. It has been installed at CSRe and will be tested in a planned experiment. With the IMS, the isochronous condition is only fulfilled strictly in a small $\mathrm{m} / \mathrm{q}-$ range. So the resolving power is not constant over the entire revolution-time spectrum. In real experiments the storage ring is tuned for the nuclei of interest, where the resolving power is the highest and it slowly decreases towards both sides of the revolution-time spectrum. Two time-of-flight detectors are planned to be installed in one of the straight sections of the CSRe. In this way, velocities of the individual stored ions can be measured in the ring. The information on the particle velocities 


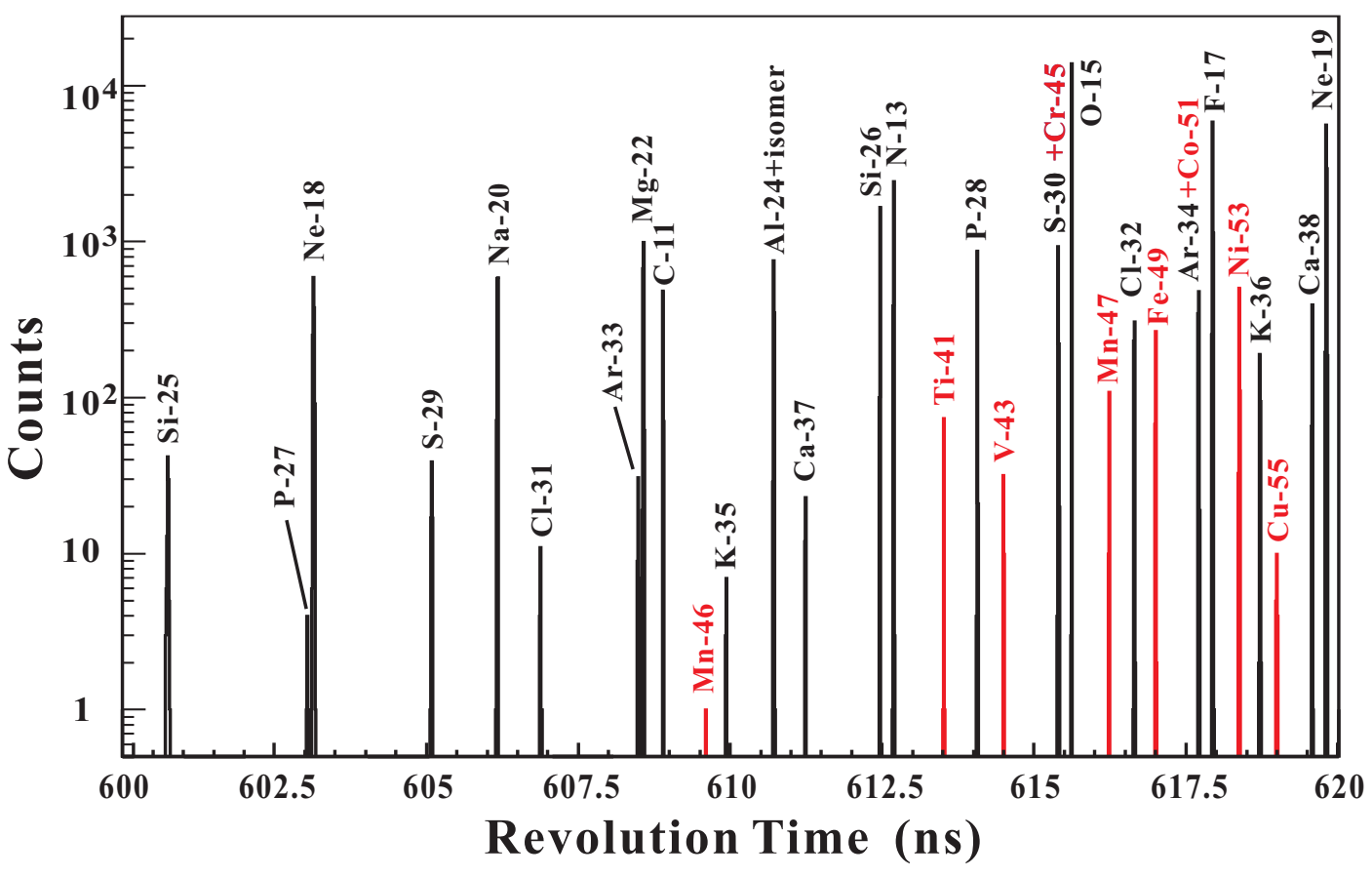

Figure 3: The revolution time spectrum obtained in ${ }^{58} \mathrm{Ni}$ experiment. The peaks with red color are nuclides of interest in this experiment.

should allow us to obtain a high resolving power for the entire revolution-time spectrum, which has an extension of $\Delta(\mathrm{m} / \mathrm{q}) /(\mathrm{m} / \mathrm{q})=10 \%$. Thus, a larger number of nuclei will be measured or used as references in a single setting of the future RIBLL2-CSRe system.

\section{References}

[1] G. Q. Xiao et al., Int. J. Mod. Phys. E18, 405 (2009).

[2] J. W. Xia et al., Nucl. Instr. and Methods A488, 11 (2002).

[3] H. S. Xu et al., Int. J. Mod. Phys. E18, 9 (2009).

[4] R. Barna et al, Nucl. Instr. Meth. A519, 610 (2004).

[5] C. Zheng et al., Chin. Phys. C 31, 1177 (2007).

[6] Z. G. Xiao et al., J. Phys. G36, 064040 (2009).

[7] X. W. Ma et al., J. Phys.: Conf. Ser. 185, 012024 (2009).

[8] Yu. A. Litvinov, Int. J. Mod. Phys. E18, 323 (2009).

[9] X.L. Tu et al., Chin. Phys. C 33(2009) 516.

[10] B. Mei et al., Nucl. Instr. Meth. A624, 109 (2010).

[11] X.L.Tu et al., Nucl. Instr. Meth. A654, 213 (2011). 
[12] X.L.Tu et al., Phys. Rev. Lett. 106 (2011) 112501.

[13] F. Nolden et al., Nucl. Instr. and Methods A 659 (2011) 69. 